\title{
EL PAPEL DE LAS UNIVERSIDADES EN LA FORMACIÓN INTEGRAL DE LOS FUTUROS PROFESIONALES
}

\author{
Claudia Sofía Blanco ${ }^{1}$ \\ Carlos Eduardo Guevara Escobar ${ }^{2}$ \\ José Pedro Zamudio Alarcón ${ }^{3}$ \\ Edgar Ferrucio Correa Coppola \\ William Ferney Cruz
}

\section{Resumen}

Las universidades nacen de la necesidad humana de permitir a sus comunidades el acceso al conocimiento, en razón a que antes de su existencia la oportunidad de acceso a éste era prácticamente nula, hoy en día los estudiantes y egresados de las universidades integran y dirigen las organizaciones que a nivel global tienen la potestad de implementar la responsabilidad social en todos los contextos; este artículo presenta los resultados de una investigación de carácter descriptivo, realizada mediante la aplicación de veintiocho encuestas en tres Instituciones de Educación Superior, ubicadas en diferentes puntos geográficos de Colombia y orientada a identificar la existencia de prácticas de responsabilidad social aplicadas por la universidad.

Los resultados de las encuestas se analizan ofreciendo su presentación en tablas de frecuencia y gráficos de barras con diferenciación de resultados por universidad mediante colores, así como el análisis de los resultados identificados y finalmente se presenta la discusión nacida del contraste entre resultados de la investigación y las propuestas incluidas en el marco teórico.

Palabras clave:Responsabilidad social universitaria, Principios éticos, Investigación para el desarrollo, Formación de ciudadanos responsables, Proyección social. 


\title{
THE ROLE OF UNIVERSITIES IN THE COMPREHENSIVE TRAINING OF FUTURE PROFESSIONALS
}

\begin{abstract}
Universities are among the oldest institutions that have endured throughout history and whose principal objective is to grant academic degrees, however, although the continuous order, every day growing concern to incorporate this training educational processes forming and develop abilities, skills, interacting with principles and values and attitudes expressed and, moreover, inform cultural frameworks, academic and disciplinary theoretical and conceptual elements and methodological foregoing in view of the loss of so many values a society with a high percentage of corruption which has great professional participation in all areas.
\end{abstract}

For the development of this article design a survey that was applied to 28 people directly related to several universities, in order to understand the role of universities in the comprehensive training of future professionals.

Keywords: University Social Responsibility, Ethical principles, Research for development, Training of responsible citizens, Social projection.

\section{Introducción}

Al interior del Área temática de investigación Gestión de las Organizaciones, se incluye el tema correspondiente a El papel de las Universidades en la formación integral de los futuros profesionales como uno de los aspectos más trascendentales en la formación integral de los dirigentes empresariales que a mediano plazo guiarán los destinos económicos y sociales del país.

En ese contexto, el objetivo de la presente investigación es el de conocer las prácticas de responsabilidad social aplicadas por tres universidades colombianas, con el fin de participar en la formación integral del futuro profesional de nuestro país.

Para lograr el objetivo planteado se elaboró un marco teórico - conceptual de temas como responsabilidad social, responsabilidad social empresarial, educación superior y responsabilidad social universitaria, se planteó la metodología y aplicaron 28 encuestas a docentes vinculados a cada una de las universidades, se realizó el tra- tamiento de la información, se presentaron los resultados gráficamente junto con su respectivo análisis y finalmente se presentaron las conclusiones logradas mediante la discusión de resultados al interior del grupo de trabajo..

\section{Desarrollo Responsabilidad Social}

La Responsabilidad Social aparece cuando una organización desarrolla una toma de conciencia compleja y holística de sí misma, de su entorno y de su propio impacto sobre su entorno. Presupone la superación de un enfoque egocéntrico, instrumental, reductor y el acceso a una conciencia organizacional global e integrada (incluye tanto a las personas como al ecosistema, tanto a los trabajadores como a los clientes y proveedores) que es capaz de «contagiar» a toda la institución (todas las personas de la organización deben poder acceder a ese nivel de conciencia) (Vallaerys, 2012). 
La RSE es la decisión de la empresa de contribuir al desarrollo sostenible, trabajando con los empleados, sus familias y la comunidad local, y también con la sociedad en su conjunto, para mejorar su calidad de vida» (World Business Council for Sustainable Development, 2006).

Según Patricia González, Directora de Responsabilidad Social Empresarial (RSE) de la Cámara de Comercio de Bogotá (CCB) La falsa creencia de que la Responsabilidad Social Empresarial es un asunto exclusivo de las grandes empresas es una de las principales barreras que afrontan las pequeñas y medianas empresas al momento de acercarse a la responsabilidad empresarial como una nueva manera de hacer negocios

Este concepto también involucra a las Universidades, instituciones que deben propender por la formación de ciudadanos responsables y capaces de transformar el mundo.

El acceso a los estudios superiores será igual para todos, en función de los méritos respectivos» de tal manera que la educación es un derecho fundamental de carácter universal y cada persona tiene la libertad de elegir que profesión estudiar de acuerdo a sus gustos, habilidades, sueños, experiencias, etc. Sin embargo, el acceso a este servicio no es tan fácil, ya que posee un costo que no es asequible para toda la población.

En la Constitución Colombiana se contempla lo siguiente: La educación es un derecho de la persona y un servicio público que tiene una función social: con ella se busca el acceso al conocimiento, a la ciencia, a la técnica, y a los demás bienes y valores de la cultura.

Para el año 2012 en nuestro país se cuenta con 58 Universidades Públicas y 160 Universidades Privadas, las cuales poseen alrededor de 1.680 .000 estudiantes a nivel de pregrado, (SNIES, 2012).
El acceso a la educación superior y a la investigación, son necesarios dentro de toda sociedad, ya que contribuyen a la erradicación de la pobreza, al desarrollo sustentable y al progreso en el alcance de las metas de desarrollo consensuadas en el ámbito internacional, tales como los Objetivos de Desarrollo del Milenio (ODM) y Educación Para Todos (EPT).

Por estos motivos, es necesario que al interior de cada Institución se creen políticas dentro y fuera del aula de clase, en cada una de las asignaturas del currículo y en las demás actividades académicas, culturales, deportivas, sociales, etc., que colaboren activamente en la formación integral de los estudiantes, que complementen los conocimientos adquiridos en cada una de las asignaturas con la realidad del país y del mundo, y que se concientice al futuro profesional del papel que va a desempeñar en la sociedad y que sus actuaciones van a influir en partes externas a su trabajo como el entorno, la comunidad, el medio ambiente, etc.

«La educación superior no sólo debe proveer de competencias sólidas al mundo presente y futuro, sino contribuir a la educación de ciudadanos éticos, comprometidos con la construcción de la paz, la defensa de los derechos humanos y los valores de la democracia».

\section{Responsabilidad Social Universitaria}

La universidad es responsable de la formación académica de todos los dirigentes de la sociedad a nivel mundial y su responsabilidad social debe empezar con una reflexión de la institución académica sobre sí misma en su entorno social, un análisis de su responsabilidad y, sobre todo, de su parte de culpabilidad en los problemas crónicos de la sociedad, dejando de pensarse como una burbuja de paz y racionalidad en medio de la tormenta (Vallaerys, 2012).

La Responsabilidad Social Universitaria exige, desde una visión holística, articular las diversas 
partes de la institución en un proyecto de promoción social de principios éticos y de desarrollo social equitativo y sostenible, para la producción y transmisión de «saberes» responsables y la formación de profesionales ciudadanos igualmente responsables (Vallaerys, 2012).

François Vallaeys, profesor de Filosofía en la PUCP y Consultor en temas de Responsabilidad Social Universitaria en la Iniciativa Interamericana de Ética, Capital Social y Desarrollo (BID) propone cuatro líneas de acción institucional como orientaciones estratégicas generales de responsabilizarían social universitaria:

- Gestión interna de la Universidad: Orientarla hacia la transformación de la Universidad en un pequeña comunidad ejemplar en Responsabilidad Social

- La docencia: Capacitar a los docentes en el enfoque de la Responsabilidad Social Universitaria y promover en las especialidades el Aprendizaje Basado en Proyectos de carácter social

- La investigación: Promover la investigación para el desarrollo

- La proyección social: Trabajar en interfaz con los departamentos de investigación y los docentes de las diversas facultades para implementar y administrar proyectos de desarrollo que puedan ser fuente de investigación aplicada y recursos didácticos para la comunidad universitaria (Vallaerys, 2012).

Arantxa Lorenzo, en su documento sobre La responsabilidad social de la universidad y el desarrollo sostenible propone como los objetivos más destacados de la responsabilidad social universitaria: «impulsar la institucionalización, generalización y sistematización de la responsabilidad social de las universidades y la sostenibilidad universitaria; Ofrecer un diagnóstico de la situación actual; Delimitar los conceptos de responsabilidad social, de desarrollo sostenible y de sostenibilidad universitaria; Promover la incorporación de estructuras e instrumentos que favorezcan la institucionalización; Favorecer los mecanismos para generar una producción de información; Elaborar recomendaciones para la incorporación de la responsabilidad social y del desarrollo sostenible en los instrumentos generales y específicos de gestión del SUE; Proponer recomendaciones para su impulso en todos los ámbitos de la vida universitaria, de manera transversal, incluyendo la comunicación interna y externa...» (Lorenzo, 2012).

Por su parte, Perla Puterman considera que la responsabilidad social aplica a todas las organizaciones y que la universidades son organizaciones, podríamos decir que como tales éstas también deberían ser responsables ante los impactos que sus actividades ocasionan en la sociedad y el medio ambiente y en consideración con sus partes interesadas y el desarrollo sostenible.

Por lo tanto y basándome una vez más en lo contenido en la Norma ISO 26000 , las universidades, como organización, deberían por principio rendir cuentas, tener un comportamiento ético, ser transparentes, respetar los derechos humanos, cumplir con la legislación nacional, respetar los intereses de las partes interesadas y cumplir con la normativa internacional de comportamiento.....» (Puterman, 2012).

\section{Metodología}

La realización de este tipo de estudio, se lleva a cabo mediante una investigación descriptiva y de campo.

El análisis de igual manera nos permite identificar, describir y analizar dicha importancia e impacto que son generadas. El alcance y el fin del estudio es medir en cierta forma el reconocimiento que activan. 


\section{Fuentes y Técnicas para la recolección de información}

Para la recolección de la información se tiene en cuenta tres aspectos básicos: Las fuentes de información, selección de instrumentos a aplicar y elaboración del instrumento.

- Fuentes de información. Para el desarrollo de la investigación se utilizan dos tipos de información (primaria y secundaria) las cuales son condensadas y analizadas para la obtención de la Discusión.

- Fuentes Primarias: Se obtuvo información básica. Cada integrante del grupo de trabajo aplicó varias encuestas a personas directamente relacionadas con una Universidad del país de donde se obtuvieron datos de la percepción del encuestado frente a las prácticas de responsabilidad social desarrolladas en la Universidad donde laboran. Las tres Universidades en las que se aplicaron las encuestas fueron la UNAD (13 encuestas), Universidad de Antioquia (8 encuestas) y UPTC (7 encuestas) para un total de 28 encuestas.

- Fuentes Secundarias: Se recopiló información de manera directa de las fuentes teóricas que respaldan la investigación.

\section{Técnicas para la recolección de Información}

El instrumento que se selecciona para la recolección de la información es la encuesta, que es la técnica basada en la interrogación directa de la fuente de información y en el posterior recuento en la muestra elegida de cada una de las diferentes respuestas obtenidas. Se elaboró un cuestionario con todas las preguntas a realizar, con preguntas cerradas, utilizándose para esta investigación el método más ampliamente utilizado para la recopilación de datos como la encuesta personal con un método de muestreo finito dada la cantidad de encuestas de la población objeto de estudio

\section{Tratamiento de la Información}

Recolectados los datos se examinaron, categorizaron y tabularon para alcanzar el fin del objeto de estudio compilándose en una tabla general en la que se integró la información de las 28 encuestas, luego se organizó mediante los procedimientos de tablas de frecuencia y gráficos de barra con diferenciación de origen por colores, para posteriormente analizar los datos.

En el proceso se utilizó el programa estadístico informático SPSS y Excel generando diferentes gráficos y tablas que permitieron interpretar de una forma lógica y eficiente la información recolectada.

\section{Objetivo de investigación}

Conocer las prácticas de responsabilidad social aplicadas por la universidad con el fin de participar en la formación integral del futuro profesional.

\section{Tipo de Investigación}

Este artículo de investigación fue de carácter descriptivo deductivo y cuantitativo

\section{Descripción de la Muestra.}

Esta investigación se enfocó en las universidades que tuvieran relación con los investigadores y de esta forma garantizar la confianza, y cordialidad que permita una información mucho más veraz y pertienente para el análisis, con el fin de cumplir la premisa «.....que desde el primer momento se cree una atmósfera de cordialidad y simpatía, un ambiente de completa libertad, sin presión, intimidación o coerción, que permita establecer lo que los autores anglosajones llaman "rapport" (comunicación positiva) entre el entrevistador y el entrevistado». (Ander Egg, 1997).

En esta investigación trabajamos con el método de muestreo finito de 28 encuestas aplicadas con un favorabilidad del $95 \%$ y un margen de error del $5 \%$ y método deductivo a partir de los resultados obtenidos por vía instrumento «cuestionario. 
Las universidades seleccionadas fueron:

Universidad Nacional Abierta y a Distancia (13), Universidad de Antioquia Seccional Magdalena Medio (8), Universidad Pedagógica y Tecnológica de Colombia UPTC (7)

\section{Resultados}

A continuación se hace un análisis de los resultados obtenidos en las encuestas, de acuerdo a cada una de las variables de gestión contempladas en este modelo, este se hace con respecto a cada universidad

\section{Pregunta No 1}

\begin{tabular}{lccccc} 
& Frecuencia & Porcentaje & $\begin{array}{c}\text { Porcentaje } \\
\text { Valido }\end{array}$ & $\begin{array}{c}\text { Porcentaje } \\
\text { Acumulado }\end{array}$ \\
\hline Total acuerdo & 6 & 21,4 & 21,4 & 21,4 \\
\hline Acuerdo & 15 & 53,6 & 53,6 & 75,0 \\
\hline Desacuerdo & 6 & 21,4 & 21,4 & 96,4 \\
\hline Total desacuerdo & 1 & 3,6 & 3,6 & 100,0 \\
\hline
\end{tabular}

Tabla No1

Gráfica No 1

PREGUNTA 1. El contenido curricular de los programas académicos en el nivel profesional que usted conoce está acordes con las necesidades del pais o de la región a la que se ofrece.

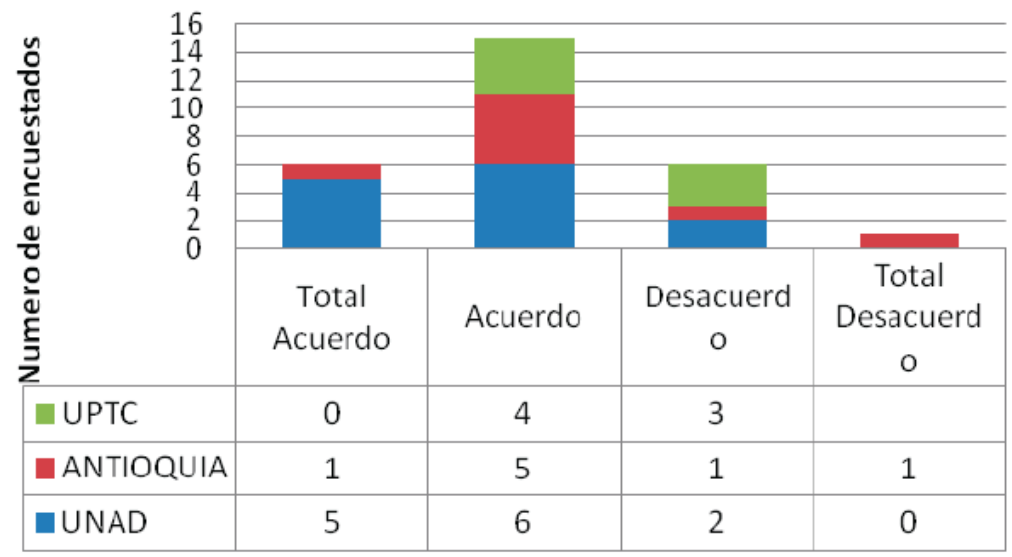

Fuente: Elaboración propia

La gráfica No 1, muestra que 21 personas encuestadas de las 28 manifiestan que los contenidos curriculares de los programas académicos a nivel profesionales (es decir un $75 \%$ ) que ofrecen las tres universidades, es- tán acordes con las necesidades del país o región, sugiriendo que estos contenidos curriculares aportan a la búsqueda de soluciones para las necesidades en las regiones en las que hacen presencia. 
Este análisis permite ver de primera mano el impacto que tiene la UNAD a nivel regional con 11 encuestas de cumplimiento, seguida de la Universidad de Antioquia con seis encuestas y finalmente la UPTC con cuatro encuestas.
Concluimos que es alta la percepción de cumplimiento en este aspecto pero deben realizarse planes de mejoramiento que permitan mantener y mejorar esta percepción al interior de cada una de nuestras universidades.

Pregunta No 2

\begin{tabular}{lccc|cc} 
& Frecuencia & Porcentaje & $\begin{array}{c}\text { Porcentaje } \\
\text { Valido }\end{array}$ & $\begin{array}{c}\text { Porcentaje } \\
\text { Acumulado }\end{array}$ \\
\hline Total acuerdo & 3 & 10,7 & 10,7 & 10,7 \\
\hline Acuerdo & 19 & 67,9 & 67,9 & 78,6 \\
\hline Desacuerdo & 5 & 17,9 & 17,9 & 96,4 \\
\hline Total desacuerdo & 1 & 3,6 & 3,6 & 100,0 \\
\hline & 28 & 100 & 100,0 & \\
\hline
\end{tabular}

Tabla No 2

Gráfica No 2

PREGUNTA 2. La gestión curricular de los programas académicos en el nivel profesional que usted conoce prevé la manera como esperan impactar en la sociedad

\begin{tabular}{|c|c|c|c|c|}
\hline 20 & & & & \\
\hline 15 & & & & \\
\hline 10 & & & & \\
\hline 5 & & & & \\
\hline ๘ & & & & \\
\hline $\begin{array}{l}\frac{1}{0} \\
\frac{1}{0} \\
\frac{0}{ \pm} \\
\text { E }\end{array}$ & $\begin{array}{c}\text { Total } \\
\text { Acuerdo }\end{array}$ & Acuerdo & $\begin{array}{c}\text { Desacuerd } \\
0\end{array}$ & $\begin{array}{c}\text { Total } \\
\text { Desacuerd } \\
0\end{array}$ \\
\hline $\bar{z}$ UPTC & 0 & 4 & 3 & 0 \\
\hline ANTIOQUIA & 0 & 6 & 1 & 1 \\
\hline UNAD & 3 & 9 & 1 & 0 \\
\hline
\end{tabular}

Fuente: Elaboración Propia

Como resultado del análisis de la gráfica No 2 , se cuenta con la certeza que 22 personas encuestadas creen que de alguna manera estas universidades logran impactar con su gestión curricular, frente a las necesidades laborales del entorno en las regiones que hace presencia.

Es importante destacar que se está de acuerdo con la analogía ofrecida por la gran mayoría que se ve expresada en el dicha tabulación, al afirmar que las universidades «deben poner la cuerda alta, muy alta, para forzar el salto del estudiante», lo cual significa, ni más ni menos, que los centros universitarios están en la obligación y el compromiso ético y social de desarrollar procesos de gestión curricular concentrados en la calidad en la formación académica y la idoneidad en el perfil de las competencias profesionales de sus graduados (impacto a la sociedad con sus programas académicos). 
Pregunta 3

\begin{tabular}{lcc|cc} 
& Frecuencia & Porcentaje & $\begin{array}{c}\text { Porcentaje } \\
\text { Valido }\end{array}$ & $\begin{array}{c}\text { Porcentaje } \\
\text { Acumulado }\end{array}$ \\
\hline Total acuerdo & 3 & 10,7 & 10,7 & 10,7 \\
\hline Acuerdo & 11 & 39,3 & 39,3 & 50,0 \\
\hline Desacuerdo & 12 & 42,9 & 42,9 & 92,9 \\
\hline Total desacuerdo & 2 & 7,1 & 7,1 & 100,0 \\
\hline & 28 & 100 & 100,0 & \\
\hline
\end{tabular}

Tabla No 3

Gráfica No 3

REGUNTA 3. La gestión curricular de los programas académicos en el nivel profesional que usted conoce cuentan con actividades de apoyo la comunidad mediante actividades de servicio social

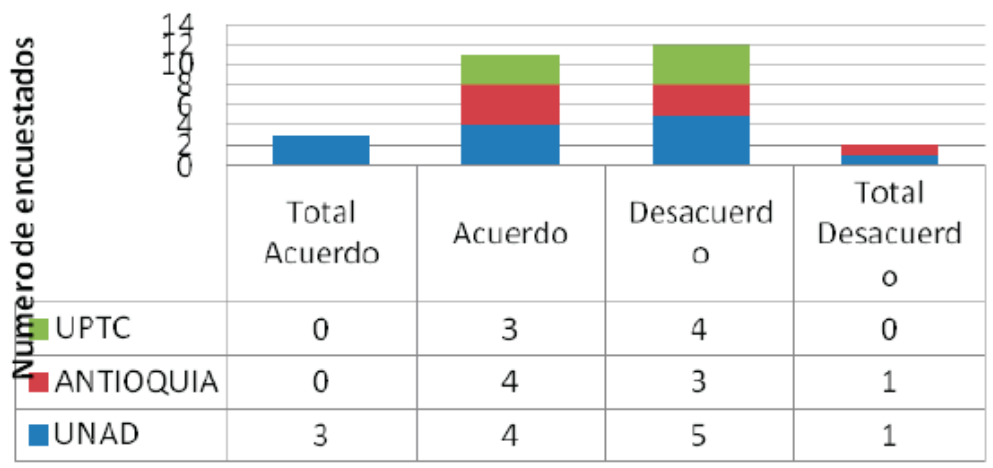

Fuente: Elaboración Propia

La gráfica No 3, demuestra que en cuanto al desarrollo de actividades sociales dirigidas a la comunidad, encontramos que tan solo un $50 \%$ de los encuestados cuentan con actividades de apoyo a la comunidad como es el servicio social, sugiriendo que teniendo en cuenta su gestión curricular se ocupan mediante actividades de servicio social por apor- tar a las necesidades de las regiones en las que hacen presencia.

Concluimos que esta es una gran oportunidad de mejoramiento que tienen las tres universidades, para mejorar su servicio social, acorde a las necesidades en las regiones en las que hace presencia cada universidad.

Pregunta 4

\begin{tabular}{lcccc} 
Frecuencia & Porcentaje & $\begin{array}{c}\text { Porcentaje } \\
\text { Valido }\end{array}$ & $\begin{array}{c}\text { Porcentaje } \\
\text { Acumulado }\end{array}$ \\
\hline Total acuerdo & 12 & 42,9 & 42,9 & 42,9 \\
Acuerdo & 14 & 50,0 & 50,0 & 92,9 \\
Desacuerdo & 1 & 3,6 & 3,6 & 96,4 \\
Total desacuerdo & 0 & 0,0 & 0,0 & 96,4 \\
& 27 & 100 & 96,4 & \\
\hline
\end{tabular}

Tabla No 4 


\section{Gráfica No 4}

PREGUNTA 4. La gestion curricular de los programas académicos en el nivel profesional que usted conoce aplican valores y principios éticos

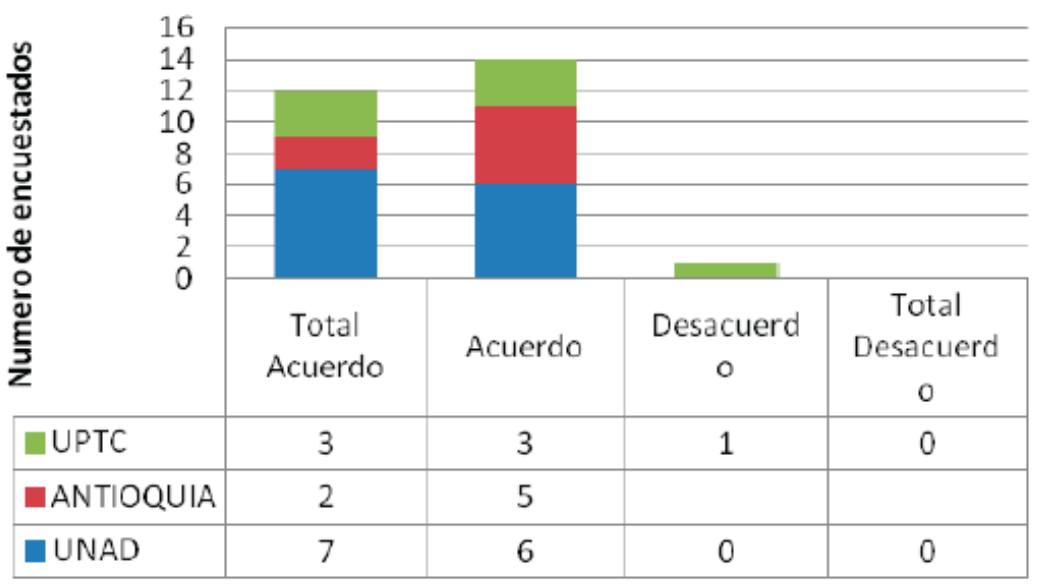

Fuente: Elaboración Propia

El resultado de la anterior gráfica demuestra que un $92 \%$ de los encuestados que corresponden a las tres universidades de estudio, consideran que la aplicación de valores y principios éticos en la gestión curricular de los programas académicos en el nivel profesional se esta cumpliendo.

De otra parte se considera que esta percepción es un paso adelante en el proceso social, sin embargo la práctica de los valores y principios éticos presentada por los medios de comunicación es razón suficiente para procurar la búsqueda de un mayor impacto social por parte de las universidades. Así entonces podemos decir que el currículum integrado y la gestión curricular a programas académicos aplicados a valores y principios éticos, se enfatiza que se está de acuerdo, porque se deduce como propuesta con la cual se contribuye con la formación de hombres y mujeres, cuyos conocimientos contemplen los contenidos suficientes de la ciencias, las disciplinas, el arte, la filosofía y la espiritualidad, como piezas flexibles que permitan mirar muy de cerca su propio espejo de intenciones, para hacer que lo nuevo que enseñe el docente, no sea un tema más, sino que esos contenidos sirvan para reflexionar en conjunto, acerca de una problemática específica y la forma de cómo puede resolverse, con la búsqueda de acertadas estrategias.

Pregunta No 5

\begin{tabular}{lcccc} 
& Frecuencia & Porcentaje & $\begin{array}{c}\text { Porcentaje } \\
\text { Valido }\end{array}$ & $\begin{array}{c}\text { Porcentaje } \\
\text { Acumulado }\end{array}$ \\
\hline Total Acuerdo & 7 & $25 \%$ & $25 \%$ & $25 \%$ \\
\hline Acuerdo & 19 & $68 \%$ & $68 \%$ & $93 \%$ \\
\hline Desacuerdo & 2 & $7 \%$ & $7 \%$ & $100 \%$ \\
\hline Total & 28 & $100 \%$ & $100 \%$ & \\
\hline
\end{tabular}

Tabla No 5 


\section{Gráfica No 5}

¿La gestión curricular de los programas académicos en el nivel profesional que usted conoce propone la responsabillidad social corporativa como parte del ejericio profesional?

\begin{tabular}{|c|c|c|c|}
\hline & & & \\
\hline 15 & & & \\
\hline 10 & & & \\
\hline 5 & & & \\
\hline षै & & & \\
\hline z & Acuerdo & Acuerdo & Desacuerdo \\
\hline UPTC & 3 & 2 & 2 \\
\hline - ANTIOQUIA & & 8 & \\
\hline —UNAD & 4 & 9 & \\
\hline
\end{tabular}

Fuente: Elaboración Propia

Como resultado del análisis de la gráfica No 5 correspondiente a la pregunta No 5 , se identifica que 27 personas encuestadas de los 28 consideran que la actual propuesta universitaria cumple con la responsabilidad social corporativa como parte del ejercicio profesional en los programas académicos en el nivel profesional que ofrecen las tres universidades encuestadas. Solamente una mínima parte equivalente al $7.0 \%$ representados por encuestados de la UPTC se encuentran inconformes.
Sin embargo, a pesar de los resultados obtenidos, por parte de los diferentes estamentos de las tres universidades frente a la responsabilidad social corporativa, se debe procurar el aseguramiento de su aplicación real en los entornos en los que ellos hacen presencia y esa es una tarea a largo plazo donde se incluya la empresa, el Estado y la Universidad.

\section{Gráfica No 6}

$¿$ Cree que la universidad cumple dentro de su responsabilidad social empresarial con la formación de profesional de profesionales íntegros que requiere el país?

\begin{tabular}{|c|c|c|c|c|}
\hline \multirow{2}{*}{ 至覞 } & \multicolumn{4}{|c|}{ 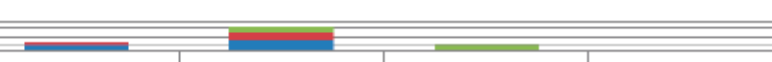 } \\
\hline & $\begin{array}{l}\text { De Total } \\
\text { Acuerdo }\end{array}$ & Acuerdo & $\begin{array}{c}\text { Desacuerd } \\
0\end{array}$ & $\begin{array}{c}\text { Total } \\
\text { Desacuerd } \\
\text { o }\end{array}$ \\
\hline U UPTC & & 4 & 3 & \\
\hline ZANTIOQUIA & 3 & 4 & 1 & \\
\hline - UNAD & 4 & 8 & & 1 \\
\hline
\end{tabular}

Fuente: Elaboración Propia 
Los datos arrojados en la gráfica No 6 nos indican que la formación integral como responsabilidad social que tiene las universidades es importante para las Universidades, objeto de estudio, de acuerdo con el análisis que se pudo realizar, 16 encuestas respondieron estar de acuerdo lo cual indica un parámetro de suma importancia frente a su formación integral y 7 están totalmente de acuerdo, concentradas en la Universidad de Antioquia y la UNAD.

Estos resultados demuestran que un $82 \%$ de los encuestados aceptan que su formación esta de acuerdo con las necesidades de profesionales íntegros que el país requiere. Es importante enfatizar que lo anterior ha significado el incremento en estrategias para potenciar el rol de cada establecimiento como eje principal en los procesos de mejora, es posible, desde este ámbito, gestionar con eficacia los procesos que ocurren al interior, específicamente los factores que dicen relación con una adecuada gestión apuntando a la responsabilidad social empresarial.

Pregunta No 7

\begin{tabular}{lcccc} 
& Frecuencia & Porcentaje & $\begin{array}{c}\text { Porcentaje } \\
\text { Valido }\end{array}$ & $\begin{array}{c}\text { Porcentaje } \\
\text { Acumulado }\end{array}$ \\
\hline De Total acuerdo & 3 & $14 \%$ & $14 \%$ & $14 \%$ \\
\hline Acuerdo & 12 & $57 \%$ & $57 \%$ & $71 \%$ \\
\hline Desacuerdo & 5 & $24 \%$ & $24 \%$ & $95 \%$ \\
\hline Total Desacuerdo & 1 & $5 \%$ & $5 \%$ & $100 \%$ \\
\hline Total & 21 & $100 \%$ & $100 \%$ & \\
\hline
\end{tabular}

Tabla No 7

Gráfica No 7

¿Se establecen los convenios necesarios entre el sector universitario y sector empresarial para lograr cumplir con la formación íntegral del profesional colombiano?

\begin{tabular}{|c|c|c|c|c|}
\hline \multirow{2}{*}{ 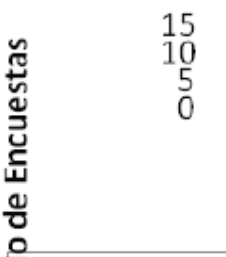 } & \multicolumn{4}{|c|}{ 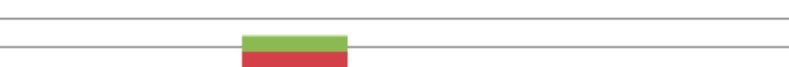 } \\
\hline & $\begin{array}{l}\text { De Total } \\
\text { Acuerdo }\end{array}$ & Acuerdo & $\begin{array}{c}\text { Desacuerd } \\
o\end{array}$ & $\begin{array}{c}\text { Total } \\
\text { Desacuerd } \\
0\end{array}$ \\
\hline z UPTC & & 3 & 5 & \\
\hline ANTIOQUIA & & 5 & & \\
\hline 口UNAD & 3 & 4 & & 1 \\
\hline
\end{tabular}

Fuente: Elaboración Propia

Los convenios entre el sector universitario y el sector empresarial son extremadamente importantes dado las actuales condiciones de globalidad y son parte esencial de la formación académica e integral del profesional colombia- no, la percepción de los encuestados frente a este comportamiento se muestra concentrado en la respuesta de acuerdo y un $14.0 \%$ están en total de acuerdo representados por la UNAD. No obstante, hay un porcentaje significativo que 
manifiesta descuerdo, equivalente a $24.0 \%$ expresado por los encuestados de la UPTC.

Lo anterior lleva a deducir que existe la necesidad de realizar convenios de beneficio mutuo entre la Universidad y el sector empresarial, da- dos los nuevos TLC firmados por nuestro país que generan la necesidad en la pequeñas y medianas empresas de vincular mano de obra capacitada, investigativa, e innovadora que sea de fácil acceso y que les permita participar en un mercado que día a día es más competitivo.

Pregunta No 8

\begin{tabular}{lcc|cc} 
& Frecuencia & Porcentaje & $\begin{array}{c}\text { Porcentaje } \\
\text { Valido }\end{array}$ & $\begin{array}{c}\text { Porcentaje } \\
\text { Acumulado }\end{array}$ \\
\hline De Total acuerdo & 4 & $14 \%$ & $14 \%$ & $14 \%$ \\
\hline Acuerdo & 10 & $36 \%$ & $36 \%$ & $50 \%$ \\
Desacuerdo & 10 & $36 \%$ & $36 \%$ & $86 \%$ \\
Total Desacuerdo & 4 & $14 \%$ & $14 \%$ & $100 \%$ \\
Total & 28 & $100 \%$ & $100 \%$ & \\
\hline \multicolumn{5}{c}{ Tabla No 8 } \\
Gráfica No 8
\end{tabular}

¿La gestión curricular de las universidades pemite la formación de profesionales altamente competitivos en el contexto internacional?

\begin{tabular}{|c|c|c|c|c|}
\hline \multirow{4}{*}{ 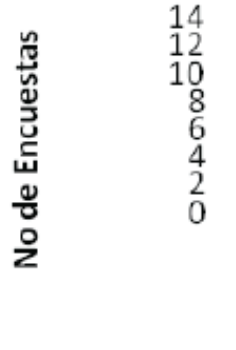 } & \multirow{3}{*}{\multicolumn{2}{|c|}{ Pre }} & \multicolumn{2}{|c|}{ 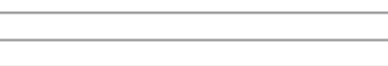 } \\
\hline & & & & \\
\hline & & & & 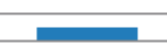 \\
\hline & $\begin{array}{l}\text { De Total } \\
\text { Acuerdo }\end{array}$ & Acuerdo & $\begin{array}{c}\text { Desacuerd } \\
0\end{array}$ & $\begin{array}{c}\text { Total } \\
\text { Desacuerd } \\
\text { o }\end{array}$ \\
\hline IUPTC & & 3 & 5 & \\
\hline ANTIOQUIA & & 5 & & \\
\hline IUNAD & 3 & 4 & & 1 \\
\hline
\end{tabular}

Fuente: Elaboración Propia

La formación profesional no debe considerarse como algo impuesto, por tal motivo se nota una alta diferencia en el consenso de la información dada, donde el punto más alto lo tiene el ítem acuerdo, es más, podría ser un momento de abstracción del mundo en donde hoy en día la competitividad es un factor altamente notable, que asegura una mínima estabilidad en un mundo global y cambiante. Hoy en día la gran parte de universidades apuntan a la formación de profesionales altamente competitivos por unificación de unos lineamientos establecidos por el ministerio de educación. 
Pregunta No 9

\begin{tabular}{lcccc} 
& Frecuencia & Porcentaje & $\begin{array}{c}\text { Porcentaje } \\
\text { Valido }\end{array}$ & $\begin{array}{c}\text { Porcentaje } \\
\text { Acumulado }\end{array}$ \\
\hline De Total acuerdo & 3 & $11 \%$ & $11 \%$ & $11 \%$ \\
\hline Acuerdo & 13 & $46 \%$ & $46 \%$ & $57 \%$ \\
\hline Desacuerdo & 11 & $39 \%$ & $39 \%$ & $96 \%$ \\
\hline Total Desacuerdo & 1 & $4 \%$ & $4 \%$ & $100 \%$ \\
\hline Total & 28 & $100 \%$ & $100 \%$ & \\
\hline
\end{tabular}

Tabla No 9

Gráfica No 9

¿La investigación y la aplicación de la tecnologia es complemento de los contenidos téoricos de su formación profesional?

\begin{tabular}{|c|c|c|c|c|}
\hline & & & & \\
\hline 12 & & & & \\
\hline 8 & & & & \\
\hline $\begin{array}{l}6 \\
4\end{array}$ & & & & \\
\hline $\begin{array}{l}4 \\
2\end{array}$ & & & & \\
\hline 0 & & & & \\
\hline & $\begin{array}{l}\text { De Total } \\
\text { Acuerdo }\end{array}$ & Acuerdo & Desacuerdo & $\begin{array}{c}\text { Total } \\
\text { Desacuerdo }\end{array}$ \\
\hline 口UPTC & 1 & 3 & 3 & \\
\hline - ANTIOQUIA & & 4 & 2 & 1 \\
\hline - UNAD & 2 & 6 & 4 & 1 \\
\hline
\end{tabular}

Fuente: Elaboración Propia

La competitividad es un factor importante que permite saber si un país va por buen camino o no en sus metas de crecimiento y aún más en este momento de globalidad y tratados TLC, de tal forma que se requiere tener capital humano altamente capacitado, es entonces que el papel que cumplen las universidades es vital durante este proceso, en contraste a esta expectativa los resultados arrojados de la anterior gráfica nos demuestra que aún falta por hacer por el conjunto de las universidades en este aspecto ya que sólo un 14 encuestados de 28 consideran que su formación los hace ser competitivos en contextos mundiales es decir tan sólo un $50 \%$.

\section{Conclusiones}

Este estudio realizado a las universidades UNAD (Universidad Nacional Abierta y a Distancia), Universidad de Antioquia y la UPTC (Universidad Pedagógica y Tecnológica de Colombia) mediante la aplicación de 28 encuestas tiene la intensión de demostrar unos resultados que nos mostraran si en verdad los contenidos curriculares de estas Universidades están acordes con las necesidades del país o de la región (en la que hacen presencia), así mismo buscamos identificar si prevé su impacto, tienen actividades de apoyo al servicio social, con valores y principios éticos, cuentan con responsabilidad 
social corporativa, formación de profesionales íntegros, los convenios entre la universidad y la empresa, la formación altamente competitiva, la investigación y tecnología, es decir si estas instituciones cumplen con todo lo anterior como instituciones públicas.

En nuestra investigación sobre la relación entre las prácticas de responsabilidad social antes enunciadas y su aplicación por parte de las universidades UNAD, Universidad de Antioquia y UPTC, con el fin de participar en la formación integral del futuro profesional; los resultados nos demuestran en primer lugar que un $75 \%$ que los contenidos curriculares de estas universidades están de acuerdo de algún modo a las necesidades de la región en que hacen presencia en el país a pesar de las diferentes modalidades que tiene en particular cada universidad. De otro lado el impacto esperado de estos contenidos están llegando en las regiones en un $78,6 \%$ es significativo pero debe permanecer y en lo posible mejorar mediante estrategias acordes con cada región, es decir las universidades de cierta manera están llegando.

Ahora con respecto al servicio social que las universidades deben prestar a las comunidades se evidencia que tan solo en un $50 \%$ esto se está haciendo la tarea en el país pero queda un 50\% que se debe cubrir con un plan de mejoramiento, para rezagar esta deuda con la comunidad.

De otro lado en este análisis observamos que en la aplicación de valores y principios éticos en los contenidos curriculares están presentes en un gran porcentaje en el conjunto de las universidades encuestadas en un $92.0 \%$, pero esto contrasta con la información recibida de los medios de comunicación de los últimos escándalos en el sector público y privado, que da a pensar que no esto se conoce pero no se aplica.
Si esto se está dando actualmente, en donde queda la Responsabilidad Social Corporativas de estas instituciones a pesar de los buenos resultados obtenidos (93.0\%) a través de las encuestas en donde se nota la falta de gestión y aplicación curricular, parece ser que no es suficiente como lo plantea François Vallaeys en donde manifiesta que "la necesidad de aplicar la responsabilidad social universitaria en todos los entornos, iniciando por la propia universidad y sus grupos de interés".

A donde queremos llegar es que la formación de profesionales íntegros no es una tarea fácil y requiere de muchos elementos no solo cognitivos, sino éticos y sociales que le den otra dimensión el que es "ser profesional integro" a pesar de los buenos resultados obtenidos en las encuestas, se requieren no solo cantidad de convenios (71\%) establecidos sino de calidad que permitan la formación de profesionales competitivos a nivel internacional (50\%), que trabajen de la mano con la investigación y desarrollo de tecnologías de punta $(57 \%)$

Se concluye con base en los resultados de esta investigación que con respecto a la Responsabilidad Social Universitaria en la formación de Profesionales Integrales se esta cumpliendo en buena parte a cabalidad en estas universidades colombianas de acuerdo a los resultados obtenidos en este estudio, si queremos profesionales competentes de alcance global es necesario revisar rigurosamente primero que la calidad de los contenidos curriculares estén acordes a las realidades de las regiones que tienen impacto en primer lugar, en segundo lugar que se afiance el lazo de la dupla academia empresa en la intención de investigación compartida y por último no olvide de su papel activo y permanente frente a las comunidades dentro del contexto que vivimos actualmente (conflicto armado). 


\section{Referencia}

Asamblea Nacional Constituyente. (2009). Constitución Política de Colombia. (1991), Bogotá - Colombia. Disponible en internet: http://www.alcaldiabogota.gov.co/ sisjur/normas/Norma1.jsp?i=4125 , con acceso $10 \mathrm{de}$ octubre de 2012

Ayuso, Cristina de la Cruz. (2008) El carácter humanizador, responsabilidad sustancial de la universidad. Alma Mater. 1 de abril de 2008.

Conferencia Mundial de Educación Superior. (2009). La nueva dinámica de la educación superior y la investigación para el cambio social y el desarrollo. Paris. Sede UNESCO. Disponible en internet: http://www.unesco.org/ education/WCHE2009/comunicado es.pdf , con acceso 10 de octubre de 2012.

Banco Interamericano de Desarrollo (2006). Iniciativa Interamericana de Capital Social, Ética y Desarrollo. Primera convocatoria del programa de apoyo a iniciativas de responsabilidad social universitaria. [En línea] 2006. [Citado el: 15 de Enero de 2008.]

De la Cuesta, Martha (2012). Responsabilidad Social Universitaria 2.0. La Coruña - España. NETBIBLO,S.L.

González, Oscar (2010). Responsabilidad Social en las Universidades Españolas. Burgos -España. Universidad de Burgos.

Lorenzo, A (2012). La responsabilidad social de la universidad y el desarrollo sostenible. Disponible en internet: http://www.responsabilidad-corporativa.es/rsu/responsabilidad-social-universitaria/documentos/item/334-laresponsabilidad-social-de-la-universidad-y-el-desarrollo-sostenible, con acceso 12 de octubre de 2012.

Ministerio de Educación, (2011). La responsabilidad social de la universidad y el desarrollo sostenible. España. Secretaría General Técnica.
MISAS, Gabriel, 2006, La educación superior en Colombia. Análisis y estrategias para su desarrollo, Bogotá, Universidad Nacional de Colombia.

ONU, (1948). Declaración Universal de derechos humanos. Paris. Asamblea General de la Naciones Unidas

Puterman, P. (2012). Avance la Responsabilidad Social Universitaria en Iberoamérica. Disponible en internet: http://www.responsabilidad-corporativa.es/rsu/ responsabilidad-social-universitaria/noticias/item/274avance-la-responsabilidad-social-universitaria-eniberoam\%C3\%A9rica, con acceso 12 de octubre de 2012.

SNIES, (2012). Información a la mano (estadísticas). Ministerio de Educación Nacional, Disponible en internet http://www.mineducacion.gov.co/sistemasdeinformacion/1735/w3-propertyname-2672.html, con acceso 04 de noviembre de 2012.

UNESCO. Declaración Mundial sobre la educación superior en el siglo XXI: visión y acción. UNESCO. París: s.n., 1998. Conferencia mundial sobre la educación superior.

Vallaeys, F. (2012). La responsabilidad social de la Universidad. Disponible en internet: http://aiesmin.unsl. edu.ar/responsabilidad.pdf , con acceso 7 de octubre de 2012.

Vallaeys, F. (2008) La Responsabilidad Social Universitaria: ¿Cómo entenderla para quererla y practicarla? Blog de ética RSU. Pontificia Universidad Católica del Perú. [En línea] 3 de Marzo de 2008. [Citado el: 15 de Mayo de 2008.]

Vessuri, Hebe. (1996).., Pertinencia de la educación superior latinoamericana a finales del siglo XX, en: Nueva Sociedad, No. 146, noviembre-diciembre. 